\title{
Kuramoto Model for Excitation-Inhibition-Based Oscillations
}

\author{
Ernest Montbrió ${ }^{1}$ and Diego Pazó ${ }^{2}$ \\ ${ }^{1}$ Center for Brain and Cognition. Department of Information and Communication Technologies, \\ Universitat Pompeu Fabra, 08018 Barcelona, Spain \\ ${ }^{2}$ Instituto de Física de Cantabria (IFCA), CSIC-Universidad de Cantabria, 39005 Santander, Spain
}

(Received 4 January 2018; revised manuscript received 10 April 2018; published 13 June 2018)

\begin{abstract}
The Kuramoto model (KM) is a theoretical paradigm for investigating the emergence of rhythmic activity in large populations of oscillators. A remarkable example of rhythmogenesis is the feedback loop between excitatory $(E)$ and inhibitory $(I)$ cells in large neuronal networks. Yet, although the $E I$-feedback mechanism plays a central role in the generation of brain oscillations, it remains unexplored whether the KM has enough biological realism to describe it. Here we derive a two-population KM that fully accounts for the onset of $E I$-based neuronal rhythms and that, as the original KM, is analytically solvable to a large extent. Our results provide a powerful theoretical tool for the analysis of large-scale neuronal oscillations.
\end{abstract}

DOI: 10.1103/PhysRevLett.120.244101

The Kuramoto model $(\mathrm{KM})$ is an idealized mathematical model for exploring the birth of collective synchronization in its most simple form. It consists of a population of heterogeneous, all-to-all coupled oscillators, and is a unique example of an exactly solvable system of nonlinear differential equations [1-5]. Yet, the KM was originally not intended as a specific description of any particular system, and finds limited applications in the modeling and analysis of natural oscillatory phenomena, see, e.g., Refs. [6-8].

An important example of collective synchronization is that of large scale neuronal oscillations [3,9]. Despite continued work using the KM to investigate neuronal rhythms (see, e.g., Refs. [10-16]), it remains unknown whether the KM actually accounts for the neuronal mechanisms resulting in such oscillations. In this Letter we derive a simple, two-population KM, that describes one of the basic mechanisms of generation of neuronal oscillations: The feedback loop between fast excitation $(E)$ and slow inhibition $(I)$ in large neuronal networks [17-20].

EI-feedback loop and EI-based oscillations.-The canonical neuronal network to model the $E I$-feedback loop consists of two interacting populations of excitatory and inhibitory neurons [21-24]. Here, we consider two populations of $N$ pulse-coupled "Winfree oscillators" [2,25-29] with phase variables $\left\{\theta_{i}^{\sigma}\right\}_{i=1, \ldots, N}$ (populations are identified by $\sigma \in\{E, I\}$ ), which evolve according to

$$
\dot{\theta}_{i}^{\sigma}=\omega_{i}^{\sigma}+\xi_{i}^{\sigma}+Q\left(\theta_{i}^{\sigma}\right)\left(K_{\sigma E} h_{E}-K_{\sigma I} h_{I}\right) .
$$

The natural frequencies $\omega_{i}^{\sigma}$ are drawn from Lorentzian distributions of half-width $\gamma$, centered at $\bar{\omega}_{\sigma}$

$$
g_{\sigma}(\omega)=(\gamma / \pi)\left[\left(\omega-\bar{\omega}_{\sigma}\right)^{2}+\gamma^{2}\right]^{-1},
$$

and $\xi_{i}^{\sigma}$ are independent, zero-mean delta-correlated noise processes of strength $D:\left\langle\xi_{i}^{\sigma}(t) \xi_{j}^{\sigma^{\prime}}\left(t^{\prime}\right)\right\rangle=2 D \delta\left(t-t^{\prime}\right) \delta_{i, j} \delta_{\sigma, \sigma^{\prime}}$.
In Eq. (1), $Q(\theta)$ is the so-called phase response curve (PRC) that determines the response of the oscillators to perturbations. Here we adopt the (infinitesimal) PRC of the theta-neuron model, $Q(\theta)=1-\cos \theta$, which is nonnegative and thus results in phase advances or delays in response to excitatory or inhibitory inputs [30-32]. Neuronal oscillators with a non-negative PRC are called Type 1, and include a broad class of neuronal models, see, e.g., Refs. [31-33]. The oscillators interact all-to-all via the mean fields

$$
h_{\sigma}=\frac{1}{N} \sum_{j=1}^{N} P\left(\theta_{j}^{\sigma}\right),
$$

which are population-averaged sums of all the pulses $P$ produced in each population. We use the family of unimodal even-symmetric functions $P(\theta)=(1-r)(1+$ $\cos \theta)\left(1-2 r \cos \theta+r^{2}\right)^{-1}$, with $\int_{-\pi}^{\pi} P(\theta) d \theta=2 \pi$ and a free parameter $r \in(-1,1)$, such that $\lim _{r \rightarrow 1} P(\theta)=2 \pi \delta(\theta)$ [28]. Expressed in words, the $j$ th oscillator in the $E$ population exerts a positive, pulselike influence $P\left(\theta_{j}^{E}\right)$ of strength $K_{E E} / N \geq 0$ to each oscillator of the $E$ population, and of strength $K_{I E} / N \geq 0$ to each oscillator of the $I$ population [similarly for the $j$ th oscillator of the $I$ population, with an explicit "-" sign in Eq. (1) corresponding to inhibition].

Figures 1(a) and 1(b) show EI-based oscillations of the mean-field quantities $h_{\sigma}$ in simulations of (a) heterogeneous and (b) noisy EI-Winfree networks, Eq. (1). The raster plots Figs. 1(c) and 1(d) show that an EI-oscillation cycle begins with the synchronous "firing" of a large cluster of phase-locked $E$ oscillators, followed by another synchronous firing of the I oscillators. Note that, to emphasize that oscillations emerge exclusively due to the interplay between fast excitatory and slow inhibitory dynamics, in 


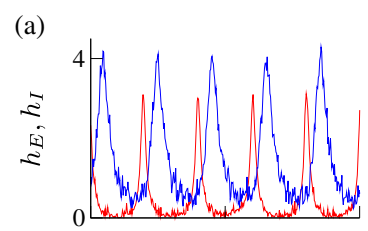

(c)

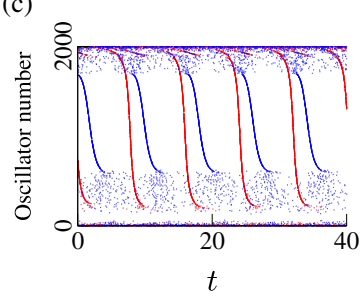

(b)

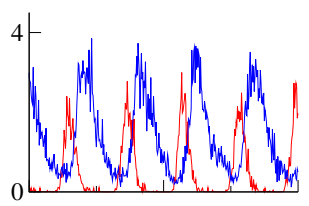

(d)

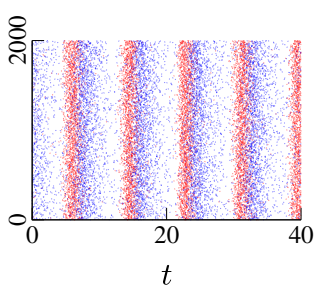

FIG. 1. EI-based oscillations in a population of $N=2000$ excitatory $(E)$ and $N=2000$ inhibitory $(I)$ Winfree oscillators, Eq. (1); with $\quad \bar{\omega}_{E}=1.5, \quad \bar{\omega}_{I}=0.5, \quad K_{E I}=K_{I E}=0.5$, $K_{E E}=K_{I I}=0$, and $r=0.99$. (a), (b) Time series of the $E$ (red) and $I$ (blue) activity-based mean fields $h_{\sigma}$. (c), (d) Raster plots: A point is plotted when an oscillator's phase reaches a multiple of $2 \pi$, which is the peak location of $P(\theta)$. In (a) and (c) frequencies are Lorentzian distributed, with $\gamma=0.1$, and $D=0$. In (b) and (d) the noise strength is $D=0.1$, and $\gamma=0$.

Fig. 1 we set the self-coupling terms to zero, $K_{E E}=$ $K_{I I}=0$, and consider $\Delta \omega \equiv \bar{\omega}_{E}-\bar{\omega}_{I}>0$. In the following we derive a two-population KM that captures the main features of the oscillations shown in Fig. 1, and that is exactly solvable to a large extent.

Excitation-inhibition Kuramoto model (EI-KM).Invoking the averaging approximation, valid for weak coupling and nearly identical oscillators [1,3], the EI-Winfree model in Eq. (1) reduces to the EI-KM [34]

$$
\begin{aligned}
\dot{\theta}_{i}^{\sigma}= & \tilde{\omega}_{i}^{\sigma}+\xi_{i}^{\sigma} \\
& -\frac{1+r}{2 N} \sum_{j=1}^{N}\left[K_{\sigma E} \cos \left(\theta_{i}^{\sigma}-\theta_{j}^{E}\right)-K_{\sigma I} \cos \left(\theta_{i}^{\sigma}-\theta_{j}^{I}\right)\right]
\end{aligned}
$$

where $\tilde{\omega}_{i}^{\sigma} \equiv \omega_{i}^{\sigma}+K_{\sigma E}-K_{\sigma I}$. There are two major differences between the EI-KM and the classical twopopulation KM broadly investigated in the literature, see, e.g., Refs. [1,35-40]. First, in the EI-KM the excitatory and inhibitory coupling constants differentially shift the natural frequencies $\tilde{\omega}_{i}^{E}$ and $\tilde{\omega}_{i}^{I}$, and this largely affects the regions of parameters where EI oscillations occur. Second, although the cosine coupling does not promote synchrony in the KM [41], the positive $(E)$ and negative $(I)$ crosscoupling terms in Eq. (4) crucially conspire to synchronize the oscillators [42]. Therefore, in the EI-KM, synchrony sets in exclusively due to the cooperative action of both the $E$ and the $I$ populations, in consonance with the EI-feedback loop mechanism. Indeed, Fig. 2 shows numerical simulations of the EI-KM in Eq. (4) using the same parameters as in Figs. 1(a) and 1(c)-except $r$, which in the $E I-K M$ is set to $r=1$, see below. Figure 2(a) displays the

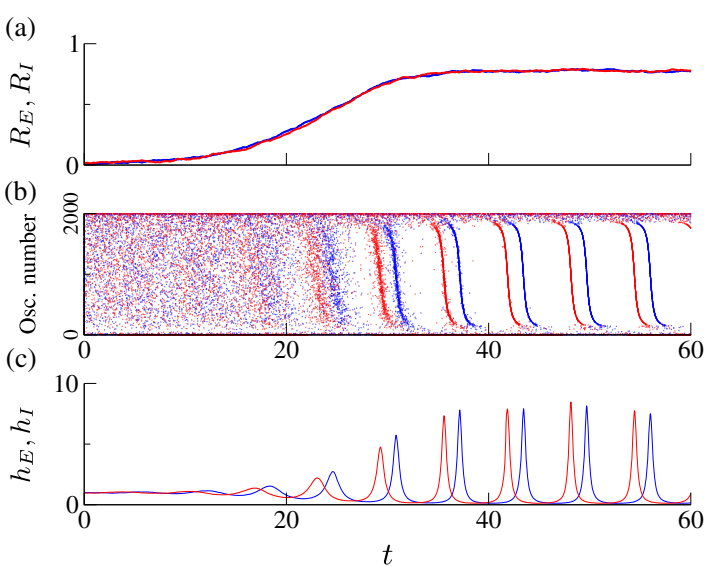

FIG. 2. EI-based oscillations in the EI-KM Eq. (4) with quenched heterogeneity and $N=2000$. (a) Amplitude of the Kuramoto order parameters, $R_{E}$ (red) and $R_{I}$ (blue); (b) raster plots; (c) mean fields obtained applying Eq. (14) to $Z_{\sigma}$; parameters are as in Figs. 1(a) and 1(c), except that here $r=1$, instead of $r=0.99$.

amplitude of the complex Kuramoto order parameters $Z_{\sigma} \equiv R_{\sigma} e^{i \Psi_{\sigma}}=N^{-1} \sum_{j=1}^{N} e^{i \theta_{j}^{\sigma}}$. At $t=0$, the amplitudes $R_{E}$ and $R_{I}$ are near zero since the initial values of all the phases are randomly distributed in the interval $[0,2 \pi)$. Then, after a brief transient, the Kuramoto order parameters converge (up to finite-size fluctuations) to uniformly rotating solutions $Z_{\sigma}(t)=R_{*} e^{i \Psi_{\sigma}(t)}$, with $0<R_{*}<1$ and $\dot{\Psi}_{\sigma}=\Omega$, signaling the onset of collective synchronization. Note that the raster plot in Fig. 2(b) shows that the cluster of $E$ oscillators precedes the cluster of $I$ oscillators, consistent with Fig. 1(c).

Finally, in the EI-KM the width of the pulses (controlled by $r$ ) influences the intensity of the cosine coupling functions. To lighten the notation, hereafter we set $r=1$ in Eq. (4), corresponding to the limit of infinitely narrow (Dirac delta) pulses - this is close to the value used in Fig. 1. The generalization of our results to general $r$ is trivial.

Analysis of the EI-KM.-Equation (4) can be efficiently analyzed in the thermodynamic limit, $N \rightarrow \infty$. To do so, the discrete sets of phases and frequencies turn into continuous variables $\left\{\theta_{i}^{\sigma}, \omega_{i}^{\sigma}\right\} \rightarrow\left\{\theta_{\sigma}, \omega_{\sigma}\right\}$, and the corresponding probability density functions $f^{\sigma}\left(\theta_{\sigma} \mid \omega_{\sigma}, t\right)$ satisfy coupled Fokker-Planck equations

$$
\partial_{t} f^{\sigma}=-\partial_{\theta_{\sigma}}\left(f^{\sigma} \dot{\theta}_{\sigma}\right)+D \partial_{\theta_{\sigma}}^{2} f^{\sigma}
$$

for which the fully incoherent state $f^{E}=f^{I}=(2 \pi)^{-1}$ is always a trivial solution [35,43]. It is convenient to introduce the Fourier expansion of $f^{\sigma}$ :

$$
f^{\sigma}(\theta \mid \omega, t)=\frac{1}{2 \pi} \sum_{l=-\infty}^{\infty} f_{l}^{\sigma}(\omega, t) e^{i l \theta}
$$

where $f_{0}^{\sigma}=1$ and $\left(f_{-l}^{\sigma}\right)^{*}=f_{l}^{\sigma}$ (the asterisk denotes complex conjugate). Thus, the Kuramoto order parameters are 


$$
Z_{\sigma}=\left(\int_{-\infty}^{\infty} f_{1}^{\sigma}(\omega, t) g_{\sigma}(\omega) d \omega\right)^{*}
$$

Substituting Eq. (6) into Eq. (5), yields two infinite sets of integro-differential equations for the Fourier modes

$$
\begin{aligned}
\dot{f}_{l}^{\sigma}= & -\left(i l \tilde{\omega}_{\sigma}+l^{2} D\right) f_{l}^{\sigma}+\frac{i l}{2} f_{l-1}^{\sigma}\left(K_{\sigma E} Z_{E}^{*}-K_{\sigma I} Z_{I}^{*}\right) \\
& +\frac{i l}{2} f_{l+1}^{\sigma}\left(K_{\sigma E} Z_{E}-K_{\sigma I} Z_{I}\right),
\end{aligned}
$$

where $\tilde{\omega}_{\sigma} \equiv \omega_{\sigma}+K_{\sigma E}-K_{\sigma I}$. The stability of the incoherent state can be analyzed by linearizing Eq. (8) [44]. To simplify the analysis, we study the case in which cross- and self-couplings are symmetric,

$$
K_{E I}=K_{I E} \equiv K, \quad K_{I I}=K_{E E} \equiv \epsilon K,
$$

and use the new parameter $\epsilon \geq 0$ as a measure of the ratio of self- to cross-coupling. Then we find that the eigenvalues determining the stability of incoherence are

$$
\lambda_{ \pm}=-\gamma-D \pm \frac{1}{2} \sqrt{K^{2}-[\Delta \omega+(\epsilon-2) K]^{2}}-i \Omega
$$

where $\Omega=\left(\bar{\omega}_{E}+\bar{\omega}_{I}\right) / 2$ is the center of the frequency distribution combining $E$ and $I$ populations. Note that parameters $\gamma$ and $D$ play identical roles in Eq. (10), as it occurs in the $\mathrm{KM}[40,43]$. Imposing $\operatorname{Re}\left(\lambda_{+}\right)=0$ in Eq. (10), we find the boundary of incoherence

$$
\left(\frac{\Delta \omega}{\gamma+D}\right)_{c}^{ \pm}=(2-\epsilon) \frac{K}{\gamma+D} \pm \sqrt{\left(\frac{K}{\gamma+D}\right)^{2}-4},
$$

which is the family of hyperbolas depicted by solid and dashed black lines in Figs. 3(a)-3(d), for increasing values of $\epsilon$. A necessary condition for the boundary Eq. (11) to exist is

$$
\frac{K}{\gamma+D} \geq 2
$$

Hence, given a certain level of heterogeneity and/or noise, synchronization sets in at large enough values of the coupling strength. This is remarkably similar to the KM [1,43], although here $K$ represents cross-, and not selfcoupling. Moreover, Eq. (12) is not a sufficient condition for synchronization in the EI-KM. If Eq. (12) is satisfied, then Eq. (11) shows that synchronization is only achieved for a particular range of values of the frequency mismatch $\Delta \omega$. The coupling ratio $\epsilon$ does not affect Eq. (12), but it critically controls the range of $\Delta \omega$ for stable incoherence: Note that when $\epsilon \leq 1$, the boundary Eq. (11) is located at positive values of $\Delta \omega$, and thus incoherence is always stable when $I$ oscillators are intrinsically faster than $E$
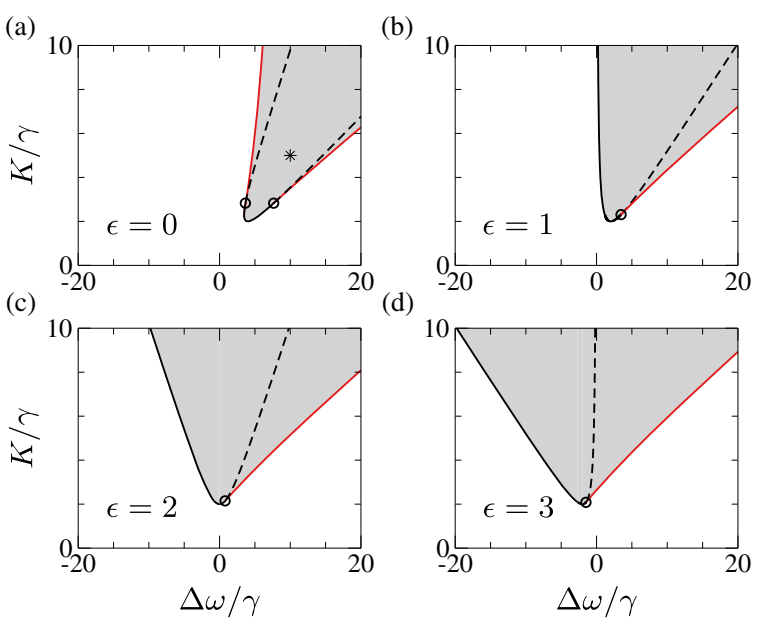

(d)

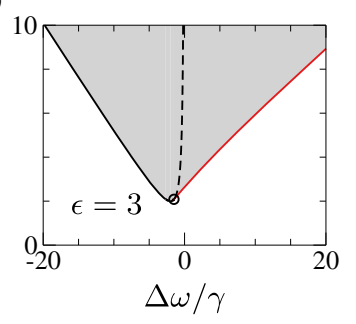

FIG. 3. Phase diagrams of the EI-KM Eq. (4) with $D=0$ and coupling constants given by Eq. (9), for (a) $\epsilon=0$, (b) $\epsilon=1$, (c) $\epsilon=2$, and (d) $\epsilon=3$. Regions of stable synchronization are highlighted in gray. Synchronization and incoherence are both stable in regions limited by black-dashed and red lines. The asterisk in (a) marks the parameter values used in Fig. 2. Black lines correspond to Eq. (11). Solid and dashed lines are separated by codimension-2 points — obtained from Eq. (19) — and indicate super- and subcritical bifurcations, respectively. Red curves indicate saddle-node bifurcations.

oscillators $(\Delta \omega<0)$, see Fig. 3. Increasing the parameter $\epsilon$ shifts the boundary, with asymptotes at $K=\Delta \omega /(3-\epsilon)$ and $K=\Delta \omega /(1-\epsilon)$, towards negative values of $\Delta \omega$. Thus, increasing the coupling ratio through $\epsilon$ provides a key ingredient for synchronizing $E I$ networks when $\bar{\omega}_{I}>\bar{\omega}_{E}$, as $I$-to- $I$ coupling slows down $I$ oscillators while $E$-to- $E$ coupling speeds up $E$ oscillators.

The synchronization region turns out to be larger than the hyperbolic boundary defined by Eq. (11), particularly for large $\epsilon$ values (see Fig. 3 for the noise-free case). The reason is that the bifurcation at Eq. (11) is often subcritical. To investigate this further, next we consider the purely heterogeneous $(D=0)$ and the purely noisy $(\gamma=0)$ cases separately, and show that the global picture is remarkably similar in both instances.

The noise-free problem is particularly simple since it can be assumed that the densities in Eq. (6) satisfy the so-called Ott-Antonsen (OA) ansatz [45,46]

$$
f_{l>1}^{\sigma}(\omega, t)=\left[f_{1}^{\sigma}(\omega, t)\right]^{l} .
$$

A first useful outcome of the OA ansatz is that it allows us to infer the mean field $h_{\sigma}$, Eq. (3), from the Kuramoto order parameter $Z_{\sigma}$, Eq. (7). Specifically, in the thermodynamic limit $h_{\sigma}(t)=\int_{-\infty}^{\infty} \int_{0}^{2 \pi} P(\theta) f^{\sigma}(\theta \mid \omega, t) g_{\sigma}(\omega) d \omega d \theta$. Then, considering $P(\theta)$ as defined above, and the heterogeneity in Eq. (2), one finds $h_{\sigma}=\operatorname{Re}\left[\left(1+Z_{\sigma}\right) /\left(1-r Z_{\sigma}\right)\right]$, see Ref. [47]. In the limit $r \rightarrow 1$, this relation reduces to 


$$
h_{\sigma}=\left(1-R_{\sigma}^{2}\right)\left(1+R_{\sigma}^{2}-2 R_{\sigma} \cos \Psi_{\sigma}\right)^{-1} .
$$

Figure 2(c) displays the mean fields $h_{\sigma}(t)$ obtained applying Eq. (14) to the Kuramoto order parameters $Z_{\sigma}(t)$ of the $E I$-KM. It can be seen that uniformly rotating solutions of the Kuramoto order parameters correspond to pulsatile oscillations of the activity-based mean fields $h_{\sigma}(t)$ [48]. Though the agreement between Figs. 1(a) and 2(c) is only qualitative, it gradually improves as parameters $\gamma$ and $\Delta \omega$ are decreased and the averaging approximation becomes more accurate [49].

A major simplification occurs assuming that $f^{\sigma}$ evolve in the so-called OA manifold, Eq. (13), as the system of Eq. (8) becomes independent of the index $l$. Then, solving the integrals in Eq. (7) by virtue of the residue theorem, we find a system of two complex-valued ordinary differential equations for $Z_{\sigma}(t)=f_{1}^{\sigma}\left(\omega=\bar{\omega}_{\sigma}-i \gamma, t\right)^{*}$

$$
\dot{Z}_{\sigma}=i\left[\hat{\tilde{\omega}}_{\sigma} Z_{\sigma}-\frac{K_{\sigma E}}{2}\left(Z_{\sigma}^{2} Z_{E}^{*}+Z_{E}\right)+\frac{K_{\sigma I}}{2}\left(Z_{\sigma}^{2} Z_{I}^{*}+Z_{I}\right)\right],
$$

with $\hat{\tilde{\omega}}_{\sigma} \equiv \bar{\omega}_{\sigma}+K_{\sigma E}-K_{\sigma I}+i \gamma$. Restricting our analysis to the case defined by Eq. (9), Eq. (15) reduces to a threedimensional system for the amplitudes $R_{\sigma}$ and the phase difference $\Phi \equiv \Psi_{E}-\Psi_{I}$. The analysis becomes further facilitated restricting to the symmetric subspace

$$
R_{E}=R_{I} \equiv R
$$

in consistency with our numerical observations, the transverse stability of the fixed points [50], and related work [51]. Hence, we analyze the planar system

$$
\begin{aligned}
& \dot{R}=R\left[-\gamma+\frac{K}{2}\left(1-R^{2}\right) \sin \Phi\right] \\
& \dot{\Phi}=\Delta \omega+K\left[\left(1+R^{2}\right) \cos \Phi-2+\epsilon\left(1-R^{2}\right)\right] .
\end{aligned}
$$

Besides the fixed point at $R_{*}=0$, corresponding to incoherence, the nontrivial fixed points of Eqs. (17) satisfy [52]

$$
\frac{\Delta \omega}{\gamma}=\left[2+\epsilon\left(R_{*}^{2}-1\right)\right] \frac{K}{\gamma} \pm\left(R_{*}^{2}+1\right) \sqrt{\frac{K^{2}}{\gamma^{2}}-\frac{4}{\left(1-R_{*}^{2}\right)^{2}}} .
$$

Figure 4(a) displays $R_{*}$ obtained from Eq. (18) for $\epsilon=0$. In this case the transitions to synchronization are hysteretic and the stable synchronized solution (solid black line) exists only in an interval of values of $\Delta \omega>0$. As the self-coupling terms are increased, Fig. 4(b) shows that the region of stable synchronization becomes broader, and invades negative values of $\Delta \omega$, see also Figs. 3(a)-3(d).
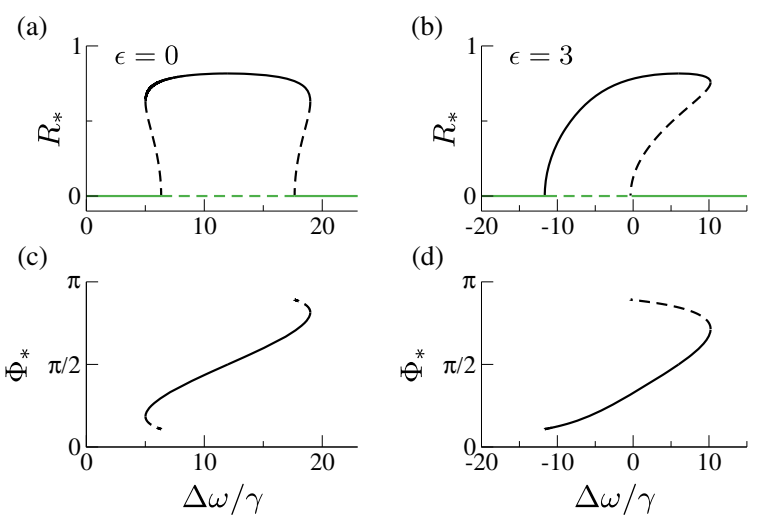

FIG. 4. Bifurcation diagrams of synchronized (black) and incoherent (green) states of Eqs. (17) for $K / \gamma=6$, obtained using Eq. (18). (a), (b) Amplitude $R_{*}$ and (c,d) phase difference $\Phi_{*}$ between the Kuramoto order parameters for (a), (c) $\epsilon=0$ and (b), (d) $\epsilon=3$.

Note that the phase difference $\Phi_{*}$ between $Z_{E}$ and $Z_{I}$ increases monotonically with $\Delta \omega$, see Figs. $4(\mathrm{c})$ and $4(\mathrm{~d})$, but lies within the interval $(0, \pi)$, and thus excitation always precedes inhibition, see also Eq. (17).

Differentiating Eq. (18) with respect to $R_{*}^{2}$ and equating the result to zero, allows us to analytically obtain the red boundaries in Fig. 3 in parametric form (not shown), corresponding to saddle-node bifurcations. As $R_{*} \rightarrow 0$, these bifurcations meet the boundaries Eq. (11) at codimension-2 points where the instabilities change from subto supercritical. The exact value of the $K$ coordinate is

$$
(K / \gamma)_{c 2}^{ \pm}=\sqrt{\left(8-2 \epsilon^{2} \mp 2 \epsilon \sqrt{8+\epsilon^{2}}\right) /\left(1-\epsilon^{2}\right)} .
$$

Substituting these values into Eq. (11) with $D=0$, we find the location of the codimension-two points represented in Fig. 3.

Finally, we have numerically verified that very similar bistability regions appear in the phase diagrams for the noisy EI-Kuramoto model Eq. (4) with identical oscillators $(D>0, \gamma=0)$. In addition, following Ref. [58], we found that the codimension-2 points of the noisy EI-KM are located at [59]

$$
(K / D)_{c 2}^{ \pm}=\sqrt{\left(12-2 \epsilon^{2} \mp 2 \epsilon \sqrt{24+\epsilon^{2}}\right) /\left(1-\epsilon^{2}\right)},
$$

which is strikingly similar to Eq. (19), but here the points lie at slightly larger $K$ values.

Conclusions.-Using the averaging approximation we derived a two-population Kuramoto model - that we call $E I$-KM-from an $E I$-network of pulse-coupled, Type 1 oscillators. The resulting EI-KM displays a transition to synchronization that has the main features of the EI-based (also known as PING, pyramidal-interneuron gamma) rhythms [17-24]: (i) Oscillations set in exclusively due 
to the cooperative action of both $\mathrm{E}$ and I populations; (ii) oscillations emerge if excitatory dynamics is faster than inhibition, irrespective of $\epsilon$. (iii) Otherwise, when inhibition is faster than excitation, strong enough selfcoupling $(\epsilon>1)$ is necessary for synchrony to occur. (iv) Excitation always precedes inhibition $\left(0<\Phi_{*}<\pi\right)$. (v) The transition between incoherence and synchronization is often hysteretic, see, e.g., Ref. [23]. While these results have been rigorously demonstrated in the EI-KM with Lorentzian heterogeneities (by means of the OA ansatz), perturbative and numerical analysis of the $E I-\mathrm{KM}$ with noise reveal the same global picture.

We acknowledge support by MINECO (Spain) under Projects No. FIS2016-74957-P, No. PSI2016-75688-P, and No. PCIN-2015-127. We also acknowledge support by the European Union's Horizon 2020 research and innovation programme under the Marie Skłodowska-Curie Grant Agreement No. 642563.

[1] Y. Kuramoto, Chemical Oscillations, Waves, and Turbulence (Springer-Verlag, Berlin, 1984).

[2] S. H. Strogatz, Physica (Amsterdam) 143D, 1 (2000).

[3] A. S. Pikovsky, M. G. Rosenblum, and J. Kurths, Synchronization, a Universal Concept in Nonlinear Sciences (Cambridge University Press, Cambridge, England, 2001).

[4] J. A. Acebrón, L. L. Bonilla, C. J. Pérez-Vicente, F. Ritort, and R. Spigler, Rev. Mod. Phys. 77, 137 (2005).

[5] A. Pikovsky and M. Rosenblum, Chaos 25, 097616 (2015).

[6] K. Wiesenfeld, P. Colet, and S. H. Strogatz, Phys. Rev. Lett. 76, 404 (1996).

[7] S. H. Strogatz, D. M. Abrams, A. McRobie, B. Eckhardt, and E. Ott, Nature (London) 438, 43 (2005).

[8] I. Z. Kiss, Y. Zhai, and J. L. Hudson, Science 296, 1676 (2002).

[9] G. Buzsáki, Rhythms of the Brain (Oxford University Press, New York, 2006).

[10] M. Breakspear, S. Heitmann, and A. Daffertshofer, Front. Hum. Neurosci. 4, 190 (2010).

[11] J. Cabral, E. Hugues, O. Sporns, and G. Deco, NeuroImage 57, 130 (2011).

[12] R. Ton, G. Deco, and A. Daffertshofer, PLoS Comput. Biol. 10, e1003736 (2014).

[13] P. Villegas, P. Moretti, and M. A. Muñoz, Sci. Rep. 4, 5990 (2014).

[14] A. Ponce-Alvarez, G. Deco, P. Hagmann, G. L. Romani, D. Mantini, and M. Corbetta, PLoS Comput. Biol. 11, e1004100 (2015).

[15] P. Sanz-Leon, S. A. Knock, A. Spiegler, and V. K. Jirsa, NeuroImage 111, 385 (2015).

[16] S. Petkoski, A. Spiegler, T. Proix, P. Aram, J.-J. Temprado, and V. K. Jirsa, Phys. Rev. E 94, 012209 (2016).

[17] M. Whittington, R. Traub, N. Kopell, B. Ermentrout, and E. Buhl, Int. J. Psychophysiol. 38, 315 (2000).

[18] P. Tiesinga and T. J. Sejnowski, Neuron 63, 727 (2009).

[19] X.-J. Wang, Physiol. Rev. 90, 1195 (2010).

[20] G. Buzsáki and X.-J. Wang, Annu. Rev. Neurosci. 35, 203 (2012).
[21] H. R. Wilson and J. D. Cowan, Biophys. J. 12, 1 (1972).

[22] N. Brunel and X.-J. Wang, J. Neurophysiology 90, 415 (2003).

[23] D. Hansel and G. Mato, Neural Comput. 15, 1 (2003).

[24] C. Boergers and N. Kopell, Neural Comput. 15, 509 (2003).

[25] A. T. Winfree, J. Theor. Biol. 16, 15 (1967).

[26] J. T. Ariaratnam and S. H. Strogatz, Phys. Rev. Lett. 86, 4278 (2001).

[27] D. Pazó and E. Montbrió, Phys. Rev. X 4, 011009 (2014).

[28] R. Gallego, E. Montbrió, and D. Pazó, Phys. Rev. E 96, 042208 (2017).

[29] C. R. Laing, Physics 7, 10 (2014).

[30] B. Ermentrout and N. Kopell, SIAM J. Appl. Math. 46, 233 (1986).

[31] B. Ermentrout, Neural Comput. 8, 979 (1996).

[32] E. M. Izhikevich, Dynamical Systems in Neuroscience (MIT Press, Cambridge, Massachusetts, 2007).

[33] E. Brown, J. Moehlis, and P. Holmes, Neural Comput. 16, 673 (2004).

[34] Phase variables in Eq. (4) correspond to slow-phase approximations of the phases in Eq. (1); See Supplemental Material at http://link.aps.org/supplemental/10.1103/ PhysRevLett.120.244101 for its derivation.

[35] H. Okuda and Y. Kuramoto, Prog. Theor. Phys. 86, 1159 (1991).

[36] E. Montbrió, J. Kurths, and B. Blasius, Phys. Rev. E 70, 056125 (2004).

[37] E. Barreto, B. Hunt, E. Ott, and P. So, Phys. Rev. E 77, 036107 (2008).

[38] D. M. Abrams, R. Mirollo, S. H. Strogatz, and D. A. Wiley, Phys. Rev. Lett. 101, 084103 (2008).

[39] Y. Kawamura, H. Nakao, K. Arai, H. Kori, and Y. Kuramoto, Chaos 20, 043110 (2010).

[40] B. Pietras, N. Deschle, and A. Daffertshofer, Phys. Rev. E 94, 052211 (2016).

[41] H. Sakaguchi and Y. Kuramoto, Prog. Theor. Phys. 76, 576 (1986).

[42] Using $\phi_{i}^{E} \equiv \theta_{i}^{E}-\pi / 2$, Eq. (4) transforms into a twopopulation model with phases $\left\{\phi_{i}^{E}\right\},\left\{\theta_{i}^{I}\right\}$, where selfinteraction functions remain the same, while cross-interaction functions become sine functions with the precise signs to favor synchrony. Hence, for the case $K_{E E}=K_{I I}=0$ considered in Fig. 2, the system reduces to a bipartite network of Kuramoto oscillators.

[43] S. H. Strogatz and R.E. Mirollo, J. Stat. Phys. 63, 613 (1991).

[44] See Supplemental Material at http://link.aps.org/ supplemental/10.1103/PhysRevLett.120.244101 for details.

[45] E. Ott and T. M. Antonsen, Chaos 18, 037113 (2008).

[46] E. Ott and T. M. Antonsen, Chaos 19, 023117 (2009).

[47] See Supplemental Material at http://link.aps.org/ supplemental/10.1103/PhysRevLett.120.244101 for details, see also Ref. [28].

[48] See Supplemental Material at http://link.aps.org/ supplemental/10.1103/PhysRevLett.120.244101 for the proof that, in the $E I-\mathrm{KM}$, the mean field $h_{\sigma}$ is linearly related with the mean firing rate of the population of oscillators, which, compared to $h_{\sigma}$, is a more natural measure of neuronal activity in neuroscience. 
[49] See Supplemental Material at http://link.aps.org/ supplemental/10.1103/PhysRevLett.120.244101 for the numerical demonstration.

[50] See Supplemental Material at http://link.aps.org/ supplemental/10.1103/PhysRevLett.120.244101 for the proof.

[51] E. A. Martens, E. Barreto, S. H. Strogatz, E. Ott, P. So, and T. M. Antonsen, Phys. Rev. E 79, 026204 (2009).

[52] In contrast with the KM with bimodal frequency distribution [1,40,51,53-56], our numerical simulations did not reveal states with time-varying $R_{\sigma}$. The same occurs in other variants of the KM, see, e.g., Ref. [57].
[53] J. D. Crawford, J. Stat. Phys. 74, 1047 (1994).

[54] L. L. Bonilla, J. C. Neu, and R. Spigler, J. Stat. Phys. 67, 313 (1992).

[55] E. Montbrió, D. Pazó, and J. Schmidt, Phys. Rev. E 74, 056201 (2006).

[56] D. Pazó and E. Montbrió, Phys. Rev. E 80, 046215 (2009).

[57] H. Hong and S. H. Strogatz, Phys. Rev. E 85, 056210 (2012).

[58] A. Pikovsky and S. Ruffo, Phys. Rev. E 59, 1633 (1999).

[59] See Supplemental Material at http://link.aps.org/ supplemental/10.1103/PhysRevLett.120.244101 for the analytical derivation. 